\title{
An Empirical Assessment of the Impact of Strategy Implementation Actions on Digital Business Strategy
}

\author{
Marc de Baat Doelman \\ Maastricht University \\ m.debaatdoelman@maastrichtuniversity.nl
}

\author{
Anant Joshi \\ Maastricht University \\ a.joshi@maastrichtuniversity.nl \\ Alexander Brüggen \\ Maastricht University \\ a.bruggen@maastrichtuniversity.nl
}

\begin{abstract}
In this paper we investigate the effect of strategy implementation actions (SIA) on the implementation of a digital business strategy (DBS). The result presented in this paper are based on an online survey with 191 responses from senior vice presidents and vice presidents of a large German manufacturing organization. Using structural equation modeling (SEM), we find a positive significant effect SIA on the implementation of a DBS. This is an important implication for academics and practitioners alike, as the actions can serve as implementation guidelines in the future.
\end{abstract}

\section{Introduction}

The purpose of this study is to investigate how the strategy implementation process needs to be conducted to ensure a successful implementation of a DBS. Ensuring a successful implementation of DBS is crucial, as technological developments alter the competitive landscape of many industries [1]. This is also confirmed by a recent study of senior executives, which suggests that $90 \%$ of executives expect great or moderate change within their industry through disruption by digital technologies [2]. According to Davenport and Westerman [3] many large organizations like GE, Lego, Nike or Procter \& Gamble therefore started digital initiatives (e.g. digitally transforming product and service offerings, embedding sensors into many products, building an extensive new software platform for the Internet of Things). The authors however find that most of these initiatives resulted in depressed stock prices, growth issues or cost and quality issues. In this context, a study by Kane et al. [2] shows that the three biggest threats associated with disruption by digital technologies are internal issues related to lack of agility or inflexible culture(19\%), market disruption related to product obsolescence or lower barriers to entry (17\%), and competitive pressure related to more intense competition and faster or new competitors (16\%). In order to address these threats, organizations need to act quickly and confidently. It is thus important for managers to formulate the right strategic answers to these developments.

One promising answer is offered by DBS, which represents a component of organizational strategy [1]. While the literature stream on business strategy is well-researched, the academic knowledge on DBS is still at an early stage. To this end, there are some studies explaining the concept of DBS and its impact on firm performance [4]. Nonetheless, what constitutes a successful implementation of DBS remains largely unexplored. As a result, examining the determinants of DBS implementation is essential for two reasons. First, the DBS field is a combination of findings from the fields of management information systems and strategic management. It tries to exploit technological advancements by incorporating them into the long-term planning of an organization with the purpose of creating and capturing value [4]. Second, the successful implementation, not its mere ideation, of a strategy is what decides whether a competitive advantage to the focal firm ensues. Even the best formulated business strategies do not necessarily guarantee competitive advantage of a business unless they can be implemented successfully [5,6]. Drawing onto these two points, it is important to understand how the combination of management information systems and strategic management influences the implementation of a strategy in order to overcome the many difficulties that lie between the ideal of strategy alignment and the reality of implementation [7]. On the one hand, the time it takes to implement a strategy often varies to a great extent $[8,9]$ and on the other hand the reported rate of failures ranges from 7 to 90 percent in literature [10]. One potential explanation is the lack of research guidelines concerning the right strategy implementation approach $[6,10]$. If we link this to the DBS implementation, it becomes evident that to bridge this gap, an inquiry into the dimensions that help to implement DBS in a successful way is warranted. Considering the lack of understanding 
about the determinants of DBS implementation, we investigate the research question of which specific SIA influence the level of implementation of a DBS. Especially, following to the theoretical framework of de Oliveira et al. [11] we are testing for the effect of unfolding, coordination, communication, control and feedback, and development of human resources policies and employee competences on the implementation of a DBS.

We gathered data within a large German manufacturing company. The company was founded more than 100 years ago, is publicly listed and has more than 100,000 employees worldwide. We carried out an online survey addressing all senior vice presidents and vice presidents. The attained overall response rate is $38.1 \%$.

Our findings can be summarized as follows. We find that all five action dimensions are significant indicators of SIA and that the SIA significantly increase the implementation level of a DBS. This paper provides several important contributions for literature and management. First, we empirically examine the impact of these dimensions on practiced strategy and provide quantitative evidence. The findings show that the proposed dimensions might serve as an important aspect in future studies on strategy implementation and DBS. Second, the study provides theoretical insights on the implementation of a DBS. That is, we examine a set of key strategic action dimensions, which are essential for the success of DBS implementation. Thereby, we address the obstacle to strategy implementation of not having a model to guide implementation efforts mentioned by Hrebiniak [5]. Third, our findings provide valuable information on the strategy implementation process for management. This can help to reduce the failure rate of implementation efforts with regards to digital business strategies in the future. Fourth, we show the effect of SIA on the business unit level and thereby follow a call for research by Brüggen, de Baat Doelman and Joshi [12].

\section{Theoretical Background and Hypotheses}

\subsection{Digital Business Strategy}

Scholars see the DBS field as a combination of management information systems and strategic management research. The most cited definition specifies DBS as an "organizational strategy formulated and executed by leveraging digital resources to create differential value" [1]. According to the authors, a DBS therefore represents the combination of an organizational- and an IT strategy. This implies that IT strategy becomes more important and has to be seen as more than a mere functional level strategy. Even though the literature stream is growing, quantitative findings remain limited. While Brüggen et al. [12] fail to find a direct effect of DBS on performance, Leischnig et al. [4] find an indirect effect through market intelligence capability, as well as through value creation and value capturing.

In order to benefit from these performance improvements, most organizations nowadays implement a DBS, as managers try to keep up with technological developments [4]. These technological developments form the new business infrastructure and affect the new organizational logic [1]. Thereby, the time component is considered crucial by Bharadwaj et al. [1], who - next to scope, scale, and sources of business value creation and value capture see speed as one key theme of DBS. The authors argue for speed as an important component, as product launches, decision making, supply chain orchestration, and network formation and adaption are rapidly accelerating in the digital context. In this view, it is thus crucial for organizations to effectively manage the strategy implementation process with regards to the implementation of a DBS. This strategy implementation process is discussed in the next sections.

\subsection{Strategy Implementation Actions}

In the field of management studies most of the work focuses on strategy formulation and neglects the implementation phase, which leaves this field underresearched [e.g. 8,11,13,14,15]. Consequently, the number of studies reporting strategy implementation guidelines, theoretical models or a clear definition to executives is limited $[11,13,14]$. This leads to executives spending most of their time on strategy formulation as they feel more comfortable in this process while neglecting the process of strategy implementation [11]. With a lack of focus from management and a missing agreed-upon and dominant framework, many implementation efforts fail [15]. According to Mankins and Steele [9] this results in companies typically only achieving an average of $63 \%$ of the financial value compared to the value previously anticipated in strategy formulation. In addition, a study by Cândido and Santos [10] investigating several studies, reports that the failure rate of implementation efforts ranges from 7 to 90 percent. As this range is tremendously wide, they conclude that it is difficult to quantify the true number of failed implementation efforts. In any case, the rate of failure is still higher than desirable. Successful implementation is however 
crucial, as even the best strategy is useless without implementation [16]. Thus, implementation expertise is pivotal for creating and maintaining a sustainable competitive advantage [13].

During strategy implementation organizations regularly face the same problems. Several studies investigated this phenomenon in order to prevent these problems in the future [e.g. 5,7,8]. Alexander [8] surveyed 93 firms from different business sectors to assess implementation problems they experienced. The author found six implementation problems which occurred in more than $60 \%$ of the surveyed firms, namely: implementation duration was longer than planned; major unplanned problems occurred during implementation; coordination of activities was not effective; competing activities and crises distracted attention; capabilities of employees were not sufficient; and training and instruction given to lower level employees were not adequate. Another study investigating problems during the implementation process has been conducted by Beer and Eisenstat [7]. The researchers collected data of the implementation process within a number of organizations. The problems most often mentioned by employees in the organizations were: top-down or laissez-faire senior management style; unclear strategy and conflicting priorities; ineffective senior management team; poor vertical communication; poor coordination across functions, businesses or borders; and inadequate down-the-line leadership skills and development. The authors conclude that these problems can be solved by some, but not all organizations. One more study on the obstacles to effective strategy implementation has been conducted by Hrebiniak [5]. The author collected data from 443 managers that were involved in strategy implementation processes. Some of the overarching issues found were the fact that managers are trained to plan, not execute. Second, top-level managers delegate the implementation efforts to lower level employees, which reduces ownership and commitment in most of the processes. Third, the implementation process takes longer than the formulation process. This makes it harder for managers to focus on and control the implementation. Fourth, execution includes more stakeholders than strategy formulation. Here, it is important for organizations to link strategic objectives with day-to-day activities as well as people's incentive structures. All in all, most strategies fail during this important phase, resulting in important resources being wasted [13]. Implementation efforts therefore require guidelines and action plans in order to prevent an unstructured approach which only relies on initiatives of motivated individuals [5].

In order to provide some guidelines or action plans, researchers have been developing various frameworks since the early 1980s. One framework, the 5 P's model, has been developed by Pryor et al. [13] and suggests that strategy implementation is reliant on the closed-loop ranging from purpose (strategic theory), principles (values and culture theory), processes (systems theories), people (behavioral theories) to performance (measurement and feedback theories). The authors thereby integrate theory and research from different business disciplines to represent the interdependencies involved during an effective implementation process in a more comprehensive and integrated structure. Another framework, the capable organization framework, has been suggested by Crittenden and Crittenden [17]. The authors argue that structural levers and managerial skills levers offer a toolkit for implementation efforts. The structural levers are actions, programs, systems, and policies. The managerial skills levers are interacting, allocating, monitoring, and organizing. The levers help identifying efforts that on the one hand facilitate formulation and implementation and on the other hand could negatively affect the implementation process [17]. One more model is the 'strategy implementation framework' created by Okumus [15]. The author established four categories, which are organized in a process-like sequence, albeit not suggesting that implementation is linear and prescriptive. The first category is strategic content and describes the strategy development process. The second category is strategic context, which differentiates between internal and external context. This category mainly deals with aspects like environmental uncertainty, organizational structure, culture and leadership. The third category describes the operational process which aggregates actions like operational planning, resource allocation, people, communication and control. The last category is referred to as outcome and investigates the results of the implementation process. According to the author, the framework helps executives and researchers to analyze the strategy implementation of past, current and future cases. Another model, the $8 \mathrm{~S}$ 's of Strategy Execution, has been developed by Higgins [18]. It is composed of the following elements: strategy and purposes, structure, systems and processes, style (leadership/management style), staff, resources, shared values (organizational culture), and strategic performance. A major problem in today's changing environment is that most organizations have difficulties aligning the aforementioned components. The model thus helps executives in focusing their attention on the relevant fields to ensure that the strategy works [18]. This is just a selective listing of strategy implementation models and should therefore not be considered exhaustive. The models clearly 
indicate that the focus in the academic literature lies on investigating the entire strategy implementation process without putting greater importance on specific aspects such as actions or required skills.

Even though the identification of the previously mentioned implementation problems and the development of implementation frameworks have improved the success rate of implementation efforts, the failure rate is still too high [10]. One promising avenue of research to improve these failure rates has been proposed by de Oliveira et al. [11]. The authors introduced an operational model for the strategy implementation process, which serves as basis for this study. In their model, strategy implementation is set up as a construct consisting of an action dimension and a results dimension. For the remainder of this paper we will focus on the model's action dimension, as putting strategies into action is one of the most complex and difficult tasks [16]. Although the action dimension has been considered to some extent in most of the aforementioned frameworks, the framework by de Oliveira et al. [11] is the first to put an emphasis on this dimension and organize all the relevant actions among conceptual dimensions. These conceptual dimensions will be presented in the next sections.

2.2.1. The effect of unfolding on strategy implementation actions. The first conceptual dimension defines the conversions of the formulated strategy into specific actions, responsibilities, and definition of goals. This promotes alignment of efforts among all stakeholders involved [11]. This dimension can be linked to the operational planning actions of the model by Okumus [15] earlier mentioned. Unfolding activities are crucial since even the best implementation efforts cannot rescue a poorly conceptualized implementation plan. If the plan is too vague, it is of limited use as responsibilities or next steps might be unclear. If it is too detailed, departments are not able to respond to changing situations quickly enough [8]. This is in line with findings by Mankins and Steele [9] who -among others- propose two important rules for successful strategy implementation related to the unfolding dimension. First, for implementation success, it is crucial to "keep it simple but make it concrete". Second, executives must clearly specify priorities. This can be linked to the specific actions, related elements and definition of goals stated in the definition above. The definition of goals is an important aspect of implementing strategies as they heavily influence the decisions made by employees. However, transforming the strategy into concrete goals is often perceived as difficult [16]. In addition, it is important to anticipate potential problems as successful implementation is characterized by preventing implementation problems in the first place [8]. Organizations should spend enough time assessing the risks of the previously mentioned implementation problems and develop a systematic process to implement a strategy. Therefore, we argue the following:

H1a: The level of unfolding activities that a business unit conducts, is a positive indicator of SIA.

2.2.2. The effect of coordination on strategy implementation actions. The second conceptual dimension defines the needed efforts of senior and middle-level management to mobilize employees and assign leaders for the implementation efforts. This improves understanding, commitment, constructive conflict resolution and cooperation [11]. This dimension can be linked to the previously mentioned obstacle of 'poor coordination across functions, businesses or borders' by Beer and Eisenstat [7], which was cited in 9 out of 12 cases in their research. In addition, the definition points out the importance of executives during the implementation process. Successful executives need to closely accompany the implementation process and spend at least as much time on it as they spend on formulating the strategy [18] instead of delegating it to lower-level employees as the study by Hrebiniak [5] shows. Another aspect that highlights managers' importance is the fact that all the implementation models mentioned earlier address the executive level [e.g. 13,15,17,18]. Overall, managers have to carefully consider their coordination activities and ensure equal involvement and understanding among all levels of the organization. Therefore, we argue the following:

$H 1 b$ : The level of unfolding activities that a business unit conducts, is a positive indicator of SIA.

2.2.3. The effect of communication on strategy implementation actions. The third conceptual dimension defines the attempts needed to disseminate information about the strategy and the implementation efforts. This includes aspects such as corporate targets, actions, responsibilities, deadlines, expected goals, results attained, and adjustments over time. The aim is to further increase understanding of the process among the workforce [11]. Many studies on strategy implementation have already emphasized the importance of communication [e.g. 7,8,14,15,16]. According to Alexander [8] CEOs in his study mentioned communication more often than any other feature as the most important aspect promoting implementation. Communication is identified as a key issue in promoting a clear understanding of the roles and responsibilities of all stakeholders during the 
implementation. Employees are thereby made aware of their tasks and stay focused on key targets despite everyday pressure [14]. The importance of this dimension becomes apparent in the previously outlined implementation obstacles and frameworks. In enhancing the understanding of strategy, the communication aspect has been found to be a major problem in most implementation processes $[9,16]$. The study by Beer and Eisenstat [7] states poor vertical communication as the most frequently mentioned "silent killer" of strategy implementation. A badly communicated strategy makes the definition of specific actions as well as adequate resource planning impossible [9]. The 'strategy implementation framework' presented earlier includes communication as a main activity during the operational process. Here, communication is seen as "the mechanisms that send formal and informal messages about the new strategy" [15]. Communication fosters strategic consensus, which describes the concept of shared understanding and commitment to strategic directives between different individuals and groups within an organization [6]. Overall, communication has to be treated as an important dimension during the implementation process as it can otherwise turn out to be a major barrier to successful strategy implementation. Therefore, we argue the following:

H1c: The level of communication activities that a business unit conducts, is a positive indicator of SIA.

2.2.4. The effect of control and feedback on strategy implementation actions. The fourth conceptual dimension defines monitoring different developments in the internal and external environment. According to de Oliveira et al. [11], this includes the internally achieved results or potential changes that may affect the implementation process. Many researchers in early management studies treated implementation as a fairly mechanistic control function [6]. Recently however less than $15 \%$ of companies regularly benchmark their business results against performance forecast on the business unit level. This might give an explanation as to why so many companies fail in implementing their strategies [9]. One important purpose of the control and feedback function is to monitor uncontrollable factors. Particularly, the uncontrollable factors in the external environment have shown to have an adverse effect on implementation, as highlighted in the aforementioned obstacles. They were identified as a problem in $60 \%$ of the strategic decision implementations [8]. In addition, this dimension helps to overcome the obstacles brought about by the fact that the implementation process takes longer than its formulation, which leads to managers losing track of the problem. Control and feedback mechanisms can be used to translate long-term needs into short-term objectives [5]. It thereby provides the feedback management needs to keep up with the external and internal environment. As this dimension can prevent a variety of problems, it has been part of all three models outlined earlier. In the $5 \mathrm{P}$ 's model it is the main driver of the performance component [13]. This component of the model helps to measure the progress of the implementation process and gives feedback to the stakeholders involved. This helps employees to understand and evaluate their own performance and informs managers on the implementation progress and on which adjustments need to be made. In the capable organization framework, the control and feedback dimension is a component in the managerial skill 'monitoring'. This skill is used to tie rewards to achievements [17]. This can help managers in directing employees' time and effort on the tasks that are beneficial for the implementation. However, this connection cannot be made with adequate precision, if organizations show deficiencies in their control and feedback dimension. Lastly, this dimension plays a pivotal role in the strategy implementation framework by Okumus [15]. On the one hand, it helps organizations to correctly interpret the strategic context (i.e. internal and external). On the other hand, it improves the operational process, as one significant element within operations is the control and feedback component. Overall, it is important to implement the right control and feedback functions in order to promote the strategy implementation process. Therefore, we argue the following:

HId: The level of control and feedback activities that a business unit conducts, is a positive indicator of

SIA.

2.2.5. The effect of development of human resources policies and employee competences on strategy implementation actions. The fifth conceptual dimension defines the joint efforts HR area with the stakeholders of the strategy implementation process. The aim is to align HR policies with the new strategy and attract the needed competences [11]. This last dimension has likewise been mentioned with regards to the problems and models outlined earlier. The study by Alexander [8] names two implementation problems, which provide a link to the development of human resources policies and employee competences. First, the difficulty that capabilities of employees involved with the implementation were insufficient. Here, the adaption of human resource policies like wage structure or hiring criteria might be an important aspect to overcome this problem. Second, the study shows that the training and instructions given to lower level 
employees were inadequate. Both "silent killers" mentioned are in line with the findings by Beer and Eisenstat [7], emphasizing that down-the-line leadership skills and development are inadequate in many organizations. The authors argue that lowerlevel managers have not been sufficiently trained to lead change associated with a new strategy. However, this lack of training cannot only be linked to lower level managers as Hrebiniak's [5] findings show. The study shows that the fact that managers are trained to plan, not execute, constitutes an essential problem. Potential solutions to address these implementation problems have already been discussed in several implementation frameworks. The 'strategy implementation framework' by Okumus [15] highlights people as a core layer in the operational process. The author states that a key enabler for a successful strategy implementation are the recruitment of relevant workforces, the acquisition and development of new skills and knowledge, the offering of adequate training activities to develop and prepare all employees involved, and the advancement of human resource policies and practices to improve the implementation of new strategies. This is in line with the staff component introduced in Higgins' [18] framework. The component highlights the prominence organizations attach to match the number and types of employees on the job with the requirements given by the implementation challenge. In short, this dimension clearly demonstrates that it is important to align HR practices and employee competences with the strategy implementation requirements in order to ensure a successful process. Therefore, we argue the following: Hle: The level of development of human resources policies and employee competences activities that a business unit conducts, is a positive indicator of SIA.

\subsection{The Effect of Strategy Implementation Actions on Digital Business Strategy Implementation}

The previous literature review points out that specific actions can have a pivotal impact on the overall success of strategy implementation efforts. Managers have to deliberately execute and guide these actions. In addition, the five dimensions indicate that strategy implementation is a multifaceted phenomenon. Organizations should apply similar efforts on all dimensions to ensure a successful strategy implementation and consider that there is a complex interplay between all dimensions [11].

According to Porter [19], an organization's corporate strategy in general follows three underlying principles. The first principle states that organizations formulate strategies through defining a set of activities, which will help them to obtain a unique and valuable market position. Organizations have to choose between different options like serving few needs of many customers, serving broad needs of few customers, or serving broad needs of many customers in a narrow market. In case of a DBS, this principle serves a comparable purpose. Organizations need to define their activities involving relationships to firms, industries, IT infrastructure, and the external environment [1]. DBS hence is not different from business strategy in the first principle. The second principle states that strategy involves deliberating and choosing between different options. Strategy needs to set a clear direction regarding decisions like revenue growth or cost savings. Equal decisions need to be reached with regards to a DBS. A study by Mithas et al. [20] compares the effect of IT investments on sales and profitability compared to other discretionary investments like advertising and R\&D expenditures. The authors find a higher effect for IT investments compared to other investments. This effect was stronger for IT investments focusing on revenue growth as compared to those focused on cost reduction. Again, this indicates that DBS has to follow Porter's [19] principles and thus needs to make compromises on important options. The third principle states that strategy aligns a company's activities. In case of DBS, this principle is of even higher importance. The example of increased speed of product launches highlights this fact. By adding a digital business dimension to products, an organization finds itself in a network with complementary products and services from other organizations (e.g. Appstore). An organization therefore needs to coordinate its activities not only within an organization but also with other organizations [1]. Once more, this showcases similarities between business strategy and DBS, as aligning the different activities remains a core principle. As the previous discussion shows that DBS follows the same principles as business strategy, we assume that SIA defined for business strategy is equally beneficial when implementing a DBS. Therefore, we argue the following:

H2: SIA positively influence the implementation level of a $D B S$.

\section{Method}

\subsection{Sample and Data Collection}

The sample consists of all senior vice presidents and vice presidents of a large German manufacturing organization $(n=502)$. The organization targets the premium segment and was founded more than 100 
years ago. It is publicly listed and conducts business in more than 140 countries. Many of its production plants lie outside of Germany and the company has more than 100,000 employees worldwide. Its yearly revenue is close to 100 billion Euros. The organization introduced its DBS two years prior to the data gathering process. Exclusively addressing the management of an organization is reasonable in this case as their expertise and knowledge is crucial in the implementation process [15].

The manufacturing industry represents a highly interesting context due to several reasons. First, most of the firms in the manufacturing industry have to tremendously adapt their product offering, especially with regards to the addition of digital components such as connectivity, location-based services or updateability. Digitalization thus transforms formerly pure-play manufacturing companies into firms that are both manufacturers and service providers. This has wide-ranging implications on core functions like product development, IT, manufacturing, logistics, marketing, sales, and after-sale. Second, large manufacturing organizations are heavily exposed to fluctuations in the world market, as well as to developments like changing regulations, customer expectations and competition. It is therefore important for them to implement strategic decisions quickly. Lastly, large manufacturing organizations provide the opportunity to obtain a large sample of test subjects across diverse departments and countries with duties ranging from production, development, human resources, finance, strategy to sales. The fact that our sample covers the whole organization is essential for testing the hypothesis stated, as implementation requires the interplay across different functional areas and activities [11]. Researchers therefore should not merely focus on specific functional areas of the organization [15] but study the implementation in the context of established organizations [17].

Many scholars emphasize the important role of managers during strategy implementation [e.g. 7, $8,9,15]$. The success of implementation efforts is strongly correlated with the time managers spend on implementation, as insufficient time and effort results in poor implementation outcomes [18]. Therefore, our sample represents a perfect fit for the hypothesis.

A structured questionnaire including 31 questions formed the principal means of data collection. In order to adequately address all managers globally, survey materials were presented in English. Pre-tests of the final survey were conducted with 15 department heads. Of the 502 managers in our final sample we attained an overall response rate of $38.1 \%$ which translates into 191 fully completed and usable responses. The main contact person in our sample organization was the vice president of strategy digitalization who helped us to increase the response rate by addressing our sample participants personally.

\subsection{Measurement and Validation}

An online survey asked our participants to report their perception regarding SIA taken, as well as their assessment of the implementation level of the DBS. As a well-accepted practice, we used seven-point Likertscale answering schemes for all questions.

SIA: We measure this concept by using the action dimension of the strategy implementation construct developed by de Oliveira et al. [11]. The authors conceive of SIA as a reflective second-order construct consisting of the five action dimensions. We removed all questions that solely addressed the activities of managers instead of the whole business unit. This is based on the pilot test, as managers were confused who they had to evaluate.

DBS: The aim of this variable is to measure the level of implementation of the DBS within individual business units. We use the measure introduced by Brüggen et al. [12].

\section{Results}

The following section presents the results using structural equation modeling (SEM). SEM is the preferred method when integrating variables that have not been directly measured (i.e. latent variables), as it analyzes multiple relationships simultaneously (incl. latent variables). In addition, SEM provides a mechanism to test for theoretically derived models against empirical data [21]. Prior to running the SEM, a confirmatory factor analysis has been conducted.

\subsection{Measurement Model}

Prior to calculating the SEM results, we evaluated the single components of the measurement model. In the first stage, a principal component analysis has been carried out to evaluate the variables directly measured by the different items and the latent variable is modeled as a measurement model and examined using SEM. The second stage will be shown in next section.

The principal component analysis has been conducted for the variables unfolding, coordination, communication, control and feedback, development of human resources policies and employee competences, and DBS implementation. The variation an item explains in a latent variable is defined by the standardized factor loadings. This value should not be less than 0.5 and preferably exceed 0.7 [21]. In 
addition, if the Eigenvalue exceeds 1, the single items can be treated as a factor. The indicator Average Variance Extracted (AVE) describes the average of the squared factor loadings and should be greater than 0.5 in order to imply acceptable convergent validity [21]. The consistency of the scale is shown by the reliability coefficient Cronbach's alpha. A lower limit for this indicator is 0.7 [21]. Altogether the variables unfolding, coordination, communication, control and feedback, development of human resources policies and employee competences, and DBS implementation show acceptable fit. Most of the factor loadings exceed the preferred level of 0.7 and all factor loadings besides one are above the lower threshold of 0.5 . In addition, the Eigenvalue of all variables is above the lower limit of 1 . The variables communication and control and feedback however do not meet all requirements. Communication scores 0.619 on the Cronbach's alpha and control and feedback scores 0.497 on AVE and 0.6563 on Cronbach's alpha. Although these values are slightly below the limit, we agreed to retain them in the model for two reasons. First, from a theoretical perspective both variables are an essential part of the latent construct. Second, the two variables fulfill all the other limits outlined earlier. For the variables unfolding, coordination, development of human resources policies and employee competences all fit indices are met. For the variable DBS implementation one factor loading and the AVE are marginally below the 0.5 limit. As the Cronbach's alpha is well above the 0.7 threshold and indicates good fit, we retain all items. Thus, all items are loaded onto a single factor for every variable.

We tested the latent variable SIA with respect to validity using the AMOS 24.0 software program [22]. The confirmatory factor analysis revealed a good fit based on the fit indicator explained in the next section.

\subsection{Structural Model}

In the second stage we calculated the SEM. Similar to the last step in the measurement model analysis, we used the AMOS 24.0 software program [22] to estimate the base model. The bootstrap method helped us to test for significance. The bootstrap procedure has been conducted with 2,000 samples and a bias-corrected confidence interval of 90 . The value of the measured variable resulted from calculating the averages of the single items used per variable. The value for the latent variable, SIA, has been calculated using AMOS. Since the distinct number of parameters to be estimated is 12 , the subject-to-parameter ratio of is 15.92 and lies above the rule of thumb of 5:1 [21]. As researchers have not found this single criterion indicating a model's validity, we report several fit indices which give a good indication and are in line with previous research. Tab. 1 shows the results of the structural equation model as well as the model fit indices. All in all, the indices indicate a good model fit. The chi-square value of 23.164 is significant at the 1 percent level ( $\mathrm{df}=9, \mathrm{p}=0.006)$ which is not optimal, but is mainly owed to the sample size. In order to remedy this shortcoming, we additionally used the CMIN/DF $\left(\chi^{2} / \mathrm{df}\right)$, as the chi-square statistic is affected by large sample sizes. Although a clear cutoff level has not been defined by researchers, an indicator ranging from 2 to 5 is generally accepted [21]. The value of 2.574 therefore meets this standard. The Root Mean Square Error of Approximation (RMSEA) is an additional measure that corrects for sample size and model complexity [23]. The index ranges from 0 to positive infinity with 0 indicating perfect model fit and appropriateness for confirmatory contexts [21]. Even though the threshold indicating good fit is still debated amongst researchers, most consider values below 0.1 acceptable [e.g. 21,23]. The RMSEA of 0.091 at hand therefore indicates good fit. In line with the RMSEA, the Goodness-of-Fit Index (GFI) is an absolute fit measure. The purpose of both measures is to calculate the fit between the specified model and the observed data. As a rule, a value greater than 0.9 indicates good model fit [21]. Since the results of the SEM report a GFI value of 0.957 , the model has strong explanatory power. The last fit indicator reported is the Comparative Fit index (CFI). This index represents the model's fit relative to an alternative baseline model and is therefore and incremental fit index. The lower threshold for the CFI is 0.9 with a preferred value of above 0.95 for a good fit indication [21]. The obtained value of 0.981 hence emphasizes good fit of our model. The structural model analysis shows that besides the $\mathrm{p}$-value, all indices indicate a good model fit. Consequently, all implications are based on a fundamental model and deemed generalizable. The final structural model is shown in Fig. 1.

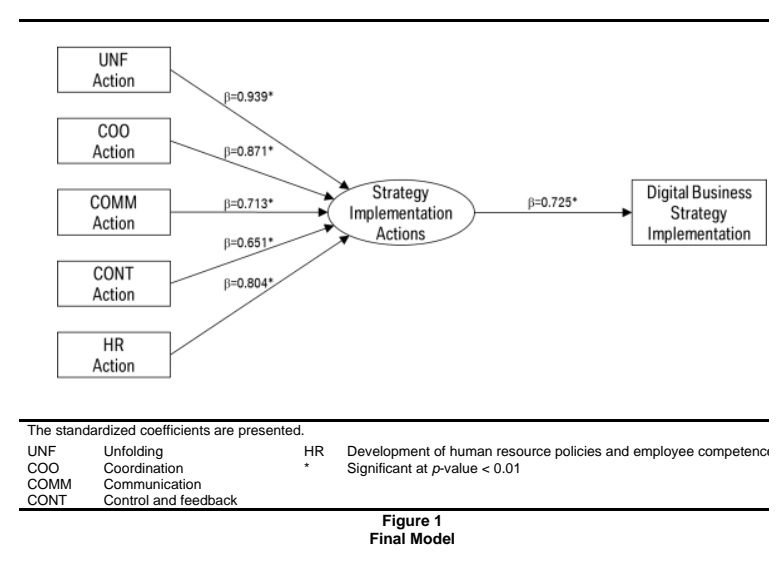




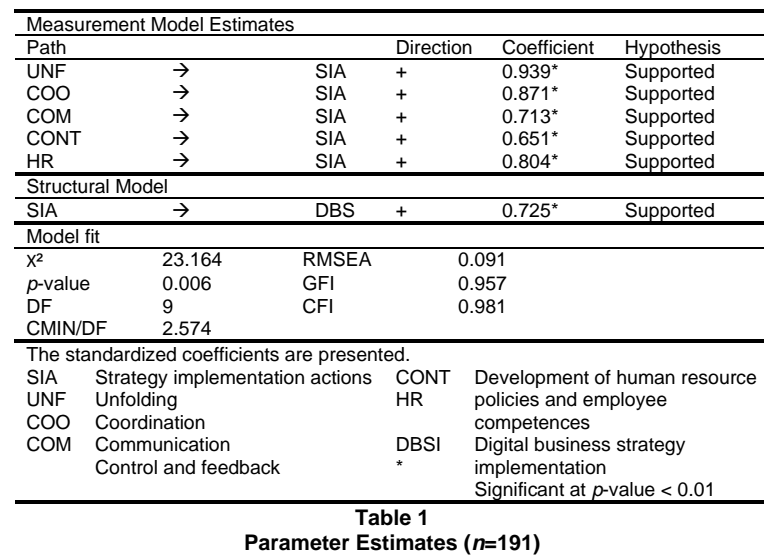

\subsection{Hypothesis Testing}

Tab. 1 presents a summary of the SEM results. Hypothesis 1a-e predicted the reflective effect of the different action dimensions on SIA. The results indicate that the weights of all second-order estimates are positive and significant. Thus, hypotheses 1a-e are significant. The strongest effect has been found for the unfolding dimension $(\beta=.939, \mathrm{p}<0.01)$ followed by coordination $(\beta=.871, \mathrm{p}<0.01)$, development of human resources policies and employee competences $(\beta=.804, \mathrm{p}<0.01)$, communication $(\beta=.713, \mathrm{p}<$ $0.01)$, and control and feedback $(\beta=.651, \mathrm{p}<0.01)$ respectively. Hypothesis 2 predicted the effect of SIA on the implementation of a DBS. The results show a positive and significant relationship between SIA and the implementation of a DBS $(\beta=.725, \mathrm{p}<0.01)$. Thus, hypothesis 2 is supported. This in line with previous literature and offers some highly appealing implications for academics and practitioners alike.

\section{Discussion and Conclusion}

Digital developments are an important topic on the agenda of researchers and executives alike. While they impact the scope of many literature streams, they are a crucial component for organizations to remain competitive. This study's aim was to answer the question of how specific SIA influence the level of implementation of a DBS. The research in both fields, strategy implementation and DBS, is still in its infancy. While DBS is a newly developing phenomenon in organizations, the field of strategy implementation lags other research fields, as most studies to date have focused on the formulation process $[6,8,13]$. In this study, we find that first, the SIA have a significant positive impact on SIA. Second, SIA have a significant positive effect on the implementation of a DBS. Responding to calls for research, this study gives guidelines on how to implement a DBS in organizations and extends the understanding of the de Oliveira et al.'s [11] model.

Our study contributes to literature in several ways. First, the findings complement the DBS field and add important findings to the fields of management information systems and strategic management. In that way, we show how findings from the two fields hold in the newly emerging field of DBS. In particular, we show how a framework from the field of strategic management can be applied to DBS. This adds quantitative evidence to the emerging field of DBS, which is an exception in a mainly theoretical and qualitatively driven field. Overall, this is an important step towards obtaining a better understanding of the DBS concept. Second, our research focuses on the under-researched area of strategy implementation instead of more common research fields like evaluating the effect of a strategy or investigating the formulation process. We tested SIA and added quantitative evidence to the field of strategy implementation, which currently remains limited. The findings show the importance of the implementation stage and indicate that researchers should pay more attention to the field, as it can help to bridge the gap between a perfectly formulated strategy and the resulting performance.

Our findings provide several contributions to management as well. First, our study tests and identifies SIA that help managers in implementing a DBS. Our findings show that strategy implementation activities should be made a core element in every strategy process. Managers have to spend more time and effort on the implementation process and refrain from solely focusing on the formulation process. Our findings thus showcase how managers can implement a DBS that addresses the threats associated with disruption through digital technologies identified by Kane et al. [2]. In addition, the findings help managers to prevent negative outcomes such as depressed stock prices, growth challenges, or cost and quality issues following the implementation of digital initiatives described by Davenport and Westerman [3]. Second, our findings offer a structured and detailed approach to management that paves the way for a successful implementation of DBS. Managers have to carefully consider the relevance of each of the implementation dimensions and subsequently tailor and apply them to their organization and business units. In the first activity, unfolding, it is important that managers translate the formulated strategy into specific actions and define goals, thus generating an implementation plan. In the second activity, coordination, it is important that managers align the efforts of all management levels to mobilize employees and establish clear responsibilities. In the third activity, 
communication, it is required that managers disseminate information regarding aspects like objectives, required actions and responsibilities. In the fourth activity, control and feedback, managers have to monitor the achieved results and observe the external and internal environment in order to adjust the plan in case this becomes necessary. In the fifth activity, development of human resource policies and employee competences, our results show that it is pivotal for managers to link the activities of the human resources department with those who implement the strategy to ensure a successful implementation.

Nonetheless, our research findings should be considered in light of several limitations. First, while we find that the SIA significantly influence the implementation level of a DBS, we did not investigate whether the sequence of implementation actions has an impact on the successful implementation of a DBS. Future studies can test the research question raised in this paper by using a time-series approach with sequential starting dates per activity. Second, all our findings are based on data from one organization. On the one hand, this has the benefit of controlling for factors like variance in quality of strategy, different levels of environmental pressure or organizational size. On the other hand, we are limited in testing for cross-industry differences, for different types of a DBS, and the effect of different external influences across industries. Future studies could address these limitations by testing the effect of SIA on the implementation of a DBS across different industries. Third, all the measures in our model are subjective and based on perception of management, as no objective measures have been made publicly available. Moreover, all the data is based on an online survey and thus possess the typical limitations. Future research can test the proposed model by using both subjective and objective measures.

\section{References}

[1] Bharadwaj, A., O. El Sawy, P. Pavlou, and N. Venkatraman. 2013. Digital business strategy: toward a next generation of insights. MIS Quarterly 37 (2): $471-$ 482.

[2] Kane, G. C., D. Palmer, A. N. Phillips, D. Kiron, and N. Buckley. 2016. Aligning the organization for its digital future. MIT Sloan Management Review, July 2016.

[3] Davenport, T. H., and G. Westerman. 2018. Why so many high-profile digital transformations fail. Harvard Business Review. April 10, 2018. https://hbr.org/2018/03/ why-so-many-high-profiledigital-transformations-fail

[4] Leischnig, A., S. Wölfl, B. Ivens, and D. Hein. 2017. From digital business strategy to market performance: insights into key concepts and processes. Proceedings of the International Conference on Information Systems (ICIS 2017), Seoul, South Korea.

[5] Hrebiniak, L. G. 2006. Obstacles to effective strategy implementation. Organizational dynamics 35 (1): 1231.

[6] Noble, C. H. 1999. The eclectic roots of strategy implementation research. Journal of Business Research 45 (2): 119-134.

[7] Beer, M., and R. A. Eisenstat. 2000. The silent killers of strategy implementation and learning. IEEE Engineering Management Review 28 (4): 35-45.

[8] Alexander, L. D. 1985. Successfully implementing strategic decisions. Long range planning 18 (3): 91-97.

[9] Mankins, M. C., and R. Steele. 2005. Turning great strategy into great performance. Harvard Business Review 83 (7/8): 64-72.

[10] Cândido, C. J., and S. P. Santos. 2015. Strategy implementation: What is the failure rate? Journal of Management \& Organization 21 (2): 237-262.

[11] de Oliveira, C. A., J. Carneiro, and F. Esteves. 2019. Conceptualizing and measuring the "strategy execution" construct. Journal of Business Research 105: 333-344.

[12] Brüggen, A., de Baat Doelman, M., \& Joshi, A. (2020). The effect of dynamic capabilities on business unit performance and digital business strategy. Working paper. Maastricht University, The Netherlands.

[13] Pryor, M. G., D. Anderson, L. A. Toombs, and J. H. Humphreys. 2007. Strategic implementation as a core competency: The 5P's model. Journal of Management Research 7 (1): 3-17.

[14] Atkinson, H. 2006. Strategy implementation: a role for the balanced scorecard? Management Decision 44 (10): 1441-1460.

[15] Okumus, F. 2003. A framework to implement strategies in organizations. Management Decision 41 (9): 871882.

[16] Aaltonen, P., and H. Ikävalko. 2002. Implementing strategies successfully. Integrated manufacturing systems 13 (6): 415-418.

[17] Crittenden, V. L., and W. F. Crittenden. 2008. Building a capable organization: The eight levers of strategy implementation. Business Horizons 51 (4), 301-309.

[18] Higgins, J. M. 2005. The eight 'S's of successful strategy execution. Journal of Change Management 5 (1): 3-13.

[19] Porter, M. E. 1996. What is strategy? Harvard Business Review 74 (6): 61-78.

[20] Mithas, S., A. Tafti, I. Bardhan, and J. M. Goh. 2012. Information technology and firm profitability: mechanisms and empirical evidence. MIS Quarterly 36 (1): 205-224.

[21] Hair, J. F. Jr., R. E. Anderson, R. L. Tatham, and W. C. Black. 2006. Multivariate data analysis. 6th ed., Upper Saddle River, NJ: Pearson Education.

[22] Arbuckle, J. L., and W. Wothke. 1999. Amos 4.0 user's guide. Chicago, IL: SmallWaters Corporation.

[23] Browne, M. W., and R. Cudeck. 1993. Alternative ways of assessing model fit. Sociological methods \& research 21 (2): 230-258. 